\title{
Regulation of Id1 expression by epigallocatechin-3-gallate and its effect on the proliferation and apoptosis of poorly differentiated AGS gastric cancer cells
}

\author{
JUN MA ${ }^{1}$, MIN SHI $^{2}$, GUANGMING LI ${ }^{3}$, NA WANG ${ }^{2}$, JUE WEI ${ }^{2}$, \\ TING WANG ${ }^{2}$, JIALI $\mathrm{MA}^{2}$ and YUGANG WANG ${ }^{2}$ \\ Departments of ${ }^{1}$ Geriatrics and ${ }^{2}$ Gastroenterology, Shanghai Changning Central Hospital, \\ Shanghai 200336; ${ }^{3}$ Department of Gastroenterology, Xinhua Hospital, \\ Shanghai Second Medical University, Shanghai 200092, P.R. China
}

Received June 7, 2013; Accepted July 17, 2013

DOI: 10.3892/ijo.2013.2043

\begin{abstract}
We investigated the inhibition of apoptosis and proliferation of poorly differentiated AGS gastric cancer cells by epigallocatechin-3-gallate (EGCG), to establish target genes for regulation by EGCG. The proliferation and apoptosis of AGS gastric cancer cells treated with EGCG were observed by cell counting kit (CCK)- 8 and flow cytometry. Differential gene expression in AGS cells treated with EGCG was screened by gene expression microarrays. Id1 gene and protein expression were determined by quantitative PCR and western blot analysis. The effect of Id1 on EGCG-induced apoptosis and cell cycle arrest of AGS cells was verified with RNAi. The proliferation and apoptosis of AGS cells treated with siRNA-Id1 was observed by CCK-8 and flow cytometry. EGCG significantly promoted apoptosis and inhibited the proliferation of AGS cells. The Id1 gene was differentially expressed in AGS cells treated with EGCG, and Id1 mRNA and protein were downregulated in AGS cells treated with EGCG, confirmed by quantitative PCR and western blot analysis. Id1 mRNA and protein were also downregulated in AGS cells treated with siRNA-Id1. The apoptosis and proliferation of AGS cells treated with siRNA-Id1 were similar to those in cells treated with EGCG. EGCG induces apoptosis and inhibits proliferation of poorly differentiated AGS gastric cancer cells, and Id1 may be one of the target genes regulated by EGCG in cancer inhibition.
\end{abstract}

Correspondence to: Professor Yu-Gang Wang, Department of Gastroenterology, Shanghai Changning Central Hospital, 1111 Xianxia Street, Shanghai 200336, P.R. China

E-mail: wang_yugang@sina.com

Abbreviations: EGCG, epigallocatechin-3-gallate; CCK-8, cell counting kit-8; PI, propidium iodide

Key words: apoptosis, epigallocatechin-3-gallate, gastric cancer, Id1, RNAi

\section{Introduction}

Gastric cancer has one of the highest incidence rates and mortality worldwide, especially in East Asia $(1,2)$. More than 400,000 new patients with gastric cancer are diagnosed in China every year. The prevalence and mortality in our country are higher than the world average (3). Most gastric cancer is adenocarcinoma, especially poorly differentiated gastric adenocarcinoma, which accounts for approximately 54\%. It features high-degree of malignancy, rapid metastasis, and poor prognosis, whereas well-differentiated gastric adenocarcinoma is characterized by slow metastasis and good prognosis. It is important to understand fully the molecular mechanism of poorly differentiated gastric cancer and effectively interfere with such mechanism. The traditional non-target chemotherapy has severe side-effects, therefore, cancer treatment and research has focused on molecular target therapy due to its high selectivity, good efficacy and low incidence of side-effects (4).

Tea is rich in polyphenols with strong antioxidant activity (5), and has a high level of epigallocatechin-3-gallate (EGCG) (6). Research on humans and animal cell models has shown that EGCG has a variety of pharmacological effects, such as strong free-radical scavenging, anti-lipid peroxidation and antiapoptotic, antiviral and antitumor activities (7). An imbalance between cell proliferation and apoptosis is one of the primary factors in the occurrence and development of tumors, therefore, proliferation inhibition and apoptosis induction of tumor cells are important methods for prevention and treatment. Many studies have confirmed that EGCG inhibits proliferation of tumor cells and induces apoptosis $(8,9)$ such as lung cancer, nasopharyngeal carcinoma, breast cancer, gastric cancer, colon cancer, prostate cancer, liver cancer, oral cancer, ovarian cancer and other malignant tumors (10-12), but its molecular mechanism is unknown and needs further study.

In the present study, we observed that EGCG induced apoptosis and inhibited proliferation of poorly differentiated AGS gastric cancer cells. The search for target genes regulated by EGCG verified that the target genes could play a role in apoptosis induction and inhibition of proliferation. 


\section{Materials and methods}

Materials. AGS cells were purchased from the Cell Resource Center of Shanghai Institutes for Biological Sciences, Chinese Academy of Sciences (Shanghai, China), Ham's F12 medium from HyClone (Logan, UT, USA), trypsin-EDTA solution and fetal bovine serum from Invitrogen (Carlsbad, CA, USA), Cell Counting Kit-8 (CCK-8) from Dojindo (Kumamoto, Japan), EGCG from Sigma (St. Louis, MO, USA), Annexin V-FITC Apoptosis Detection Kit and FACSCalibur Flow Cytometer from BD Pharmingen (San Diego, CA, USA), BioTek microplate reader from BioTek (Winooski, VT, USA) with primer designed by Shanghai Sangon Biotech Co. Ltd., Illumina BeadChip HumanHT-12_V4 from Illumina (San Diego, CA, USA), ON-TARGETplus SMARTpool siRNA targeting Id1 kit from Dharmacon (Waltham, MA, USA), Silencer siRNA Transfection II kit from Ambion (Carlsbad, CA, USA), Agarose I from Amresco (Solon, OH, USA), RNeasy mini kit from Qiagen (Dusseldorf, Germany), Reverse Transcription System from Promega (Fitchburg, WI, USA), SYBR Premix Ex Taq from Takara (Kyoto, Japan), ABI prism 7300 PCR from ABI (Carlsbad, CA, USA), Amersham ECL plus Western Blotting Detection System from GE Healthcare (Little Chalfont, UK), Pierce BCA Protein Assay Kit from Thermo Scientific (Waltham, MA, USA), 8453 UV-visible Spectroscopy System from Agilent (Santa Clara, CA, USA), inverted fluorescence microscope IX51 from Olympus (Tokyo, Japan), and Id1 antibody from Epitomics (Burlingame, CA, USA).

CCK-8 experiment. Poorly differentiated AGS cells were cultured in Ham's F12 medium $+10 \%$ FBS for $24 \mathrm{~h}$ and divided into 7 groups ( 3 holes in each group). The medium in the holes was substituted with complete medium with final concentrations of $0,20,40,60,80,120$ and $240 \mu \mathrm{g} / \mathrm{ml}$ ESPS. The proliferation inhibition rate was calculated as follows: (control - administration)/(control - background) x 100\%. Control: cells, culture medium, DMSO and CCK-8; administration: cells, culture medium and different concentrations of EGCG and CCK-8; background: only medium and CCK-8.

To verify Id1 gene and protein expression in AGS gastric cancer cells treated with EGCG and siRNA-Id1, $1 \times 10^{6}$ cells were incubated in $12100-\mathrm{mm}$ culture dishes containing $10 \mathrm{ml}$ complete medium. The medium in 6 culture dishes was substituted with complete medium containing $0,20,40,60$, 80 or $100 \mu \mathrm{g} / \mathrm{ml} \mathrm{EGCG}$, and the medium in another 6 culture dishes was substituted with medium containing $0,40,60,80$ or $100 \mathrm{nM}$ siRNA-Id1, and $100 \mathrm{nM}$ control siRNA. The complete medium was incubated in $5 \% \mathrm{CO}_{2}$ at $37^{\circ} \mathrm{C}$ for $72 \mathrm{~h}$, followed by addition of CCK-8 solution in the proportion $10 \mu \mathrm{l} / 100 \mu \mathrm{l}$, which was allowed to stand at $37^{\circ} \mathrm{C}$ for $1 \mathrm{~h}$. Absorbance was read at $450 \mathrm{~nm}$ wavelength with a microplate reader.

Gene expression microarray. AGS cells $\left(3 \times 10^{6}\right)$ were incubated in 3 100-mm culture dishes containing $10 \mathrm{ml}$ complete medium. Twenty-four hours later, when all the cells were normal, the medium was substituted with complete medium containing $80 \mu \mathrm{g} / \mathrm{ml}$ EGCG. All treatments were carried out in triplicate. Cells were collected to separate RNA, and total RNA in all samples were extracted and inspected for quality in accordance with Qiagen kit instructions. Qualified total RNA was labeled fluorescently using the Ambion Illumina RNA amplification kit from Illumina, and cRNA was hybridized with Illumina BeadChip HumanHT-12_V4 chip after linear amplification. The film was developed, the hybridization results were scanned, and the relevant data were analyzed and normalized by the Average method. The screening criteria for the differentially expressed genes were as follows: any effective gene either in the experiment group or control group $(\mathrm{P}<0.05)$ with the diffscore value in experiment group $<13$ or $>13$, and the fold-change $>2$ of the difference.

Knockdown of Idl transcripts using siRNA transfection. We used ON-TARGETplus SMARTpool siRNA targeting Id1 (50 nM, L-005051-00-0050, Dharmacon) and Silencer siRNA Transfection II kit (Ambion) in KO-DMEM medium, according to the manufacturer's instructions. Non-targeting siRNA (Ambion) was used as a negative control. The ON-TARGETplus SMARTpool siRNA reagent and at least 3 of 4 individual siRNAs silenced target gene expression by at least $75 \%$ at the mRNA level when used under optimal delivery conditions (confirmed using a validated control siRNA). Silencing was monitored at the mRNA level $24-48 \mathrm{~h}$ after transfection using $100 \mathrm{nM}$ siRNA.

Apoptosis and cell cycle analysis with flow cytometry. AGS cells were digested with trypsin-EDTA into single-cell suspensions and then collected. The resuspended cells $\left(1 \times 10^{5}\right)$ were centrifuged at 1,000 rpm for $5 \mathrm{~min}$ to remove the supernatant, and the cells were resuspended in $100 \mu \mathrm{l}$ Annexin V binding solution and transferred into a $5-\mathrm{ml}$ culture tube. Annexin V-FITC and propidium iodide (PI) $(5 \mu \mathrm{l})$ was added to the solution, and incubated in the dark at $20-25^{\circ} \mathrm{C}$ for $15 \mathrm{~min}$, followed by addition of $400 \mu \mathrm{l}$ Annexin $\mathrm{V}$ binding solution for flow cytometry. Annexin V-FITC has green fluorescence and PI has red fluorescence. The wavelength of light excited by the flow cytometer was $488 \mathrm{~nm}$. FITC fluorescence was detected with a band-pass filter of $515 \mathrm{~nm}$ wavelength and PI fluorescence was detected with a filter of $>560 \mathrm{~nm}$. The cell pellet was added to $1 \mathrm{ml}$ precooled $70 \%$ ethanol, fixed at $4^{\circ} \mathrm{C}$ overnight, washed with PBS twice, centrifuged at 1,000 rpm for $5 \mathrm{~min}$, resuspended in $0.5 \mathrm{ml}$ PBS containing $50 \mu \mathrm{g} / \mathrm{ml} \mathrm{PI}$ and $100 \mu \mathrm{g} / \mathrm{ml} \mathrm{RNase} \mathrm{A}$, and incubated in the dark at $37^{\circ} \mathrm{C}$ for $30 \mathrm{~min}$ to determine the cell cycle with a flow cytometer according to standard procedures. The result was analyzed with a cycle meter and ModFit software.

Real-time RT-PCR. Real-time RT-PCR was carried out after treatment of AGS cells. Total RNA was extracted from all samples, quantified, and reverse transcribed into cDNAs according to the instructions of the RNeasy mini kit (Qiagen). Real-time RT-PCR was carried out according to the instructions of the kit of the Reverse Transcription System (Promega). Target gene primer sequences are shown in Table II. Reaction conditions were as follows: $95^{\circ} \mathrm{C}$ for $30 \mathrm{sec} ; 95^{\circ} \mathrm{C}$ for $15 \mathrm{sec}$, and $62^{\circ} \mathrm{C}$ for $34 \mathrm{sec}$ ( 40 cycles). We used the $2^{-\Delta \Lambda} \mathrm{Ct}$ method for calculating the relative expression levels of target genes.

Western blot analysis. Western blot analysis was carried out after drug treatment. The cells were incubated with $5 \% \mathrm{CO}_{2}$ 


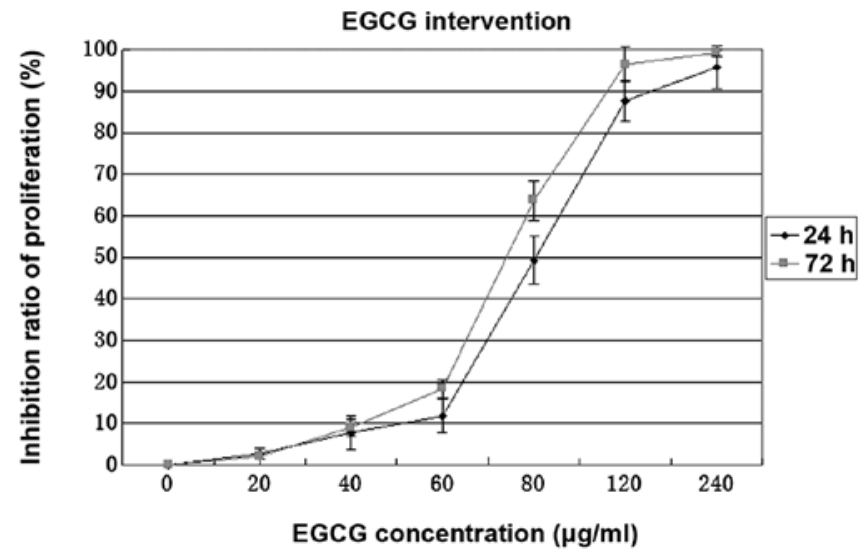

Figure 1. The CCK-8 experiment showed the effect of different concentrations of EGCG on inhibition of AGS cell proliferation. Inhibition rates with different concentrations of EGCG were significantly different $(\mathrm{P}<0.01)$. EGCG inhibited AGS cell proliferation in a concentration-dependent manner. Proliferation was significantly inhibited by $80 \mu \mathrm{g} / \mathrm{ml}$ EGCG, and the rates of inhibition of cells treated with the same concentration of EGCG for 24 and $72 \mathrm{~h}$ were not significantly different $(\mathrm{P}>0.05)$.

at $37^{\circ} \mathrm{C}$ for $48 \mathrm{~h}$, collected after digestion with pancreatin, washed twice with PBS, centrifuged at 2,000 rpm for $5 \mathrm{~min}$ to remove the supernatant, and placed on ice for lysis. The proteins were quantified by the bicinchoninic acid (BCA) method. SDS-PAGE, membrane transfer, immunoreactivity and gel electrophoresis image analysis were performed for the target genes.

Statistical analysis. The data were analyzed with SPSS 13.0 statistical software (SPSS Inc., Chicago, IL, USA), and expressed as the mean \pm SD. Multiple groups were analyzed with one-way analysis of variance, pairwise comparison was conducted by using the least significant difference (LSD) $\mathrm{t}$-test, and different groups were compared using a t-test. $\mathrm{P}<0.05$ was statistically significant.

\section{Results}

Inhibition of AGS cell proliferation and growth by EGCG. The CCK-8 experiment and cell morphological observation showed that EGCG inhibited proliferation of human gastric cancer cells in a concentration-dependent manner (Fig. 1, $\mathrm{P}<0.01)$. Proliferation of AGS cells treated with $80 \mu \mathrm{g} / \mathrm{ml}$ EGCG was significantly inhibited and the inhibition rate did not differ significantly after 24 and $72 \mathrm{~h}$ treatment (Fig. 1, $\mathrm{P}>0.05$ ).

Selection of differentially expressed genes with gene expression microarray. There were 54 differentially expressed genes when comparing EGCG-treated and normal control cells, with 37 upregulated genes of diffscore value $>13$ and 17 downregulated genes with diffscore value $<-13$ (Table I). One differentially expressed gene, Id1, in AGS gastric cancer cells before and after treatment with EGCG was screened out, and was verified subsequently (Table I). The genes were 
Table I. Real-time RT-PCR primers.

\begin{tabular}{ccc}
\hline SN & Gene & Primer sequence (5' to 3') \\
\hline 1 & $\beta$-actin & F: 5'TGGAGAAAATCTGGCACCA3' \\
& & R:5'CAGGCGTACAGGGATAGCAC3' \\
2 & Id1 & F: 5'ACGACATGAACGGCTGTTACTCAC3' \\
& & R:5'CTCCAACTGAAGGTCCCTGATGTAG3'
\end{tabular}

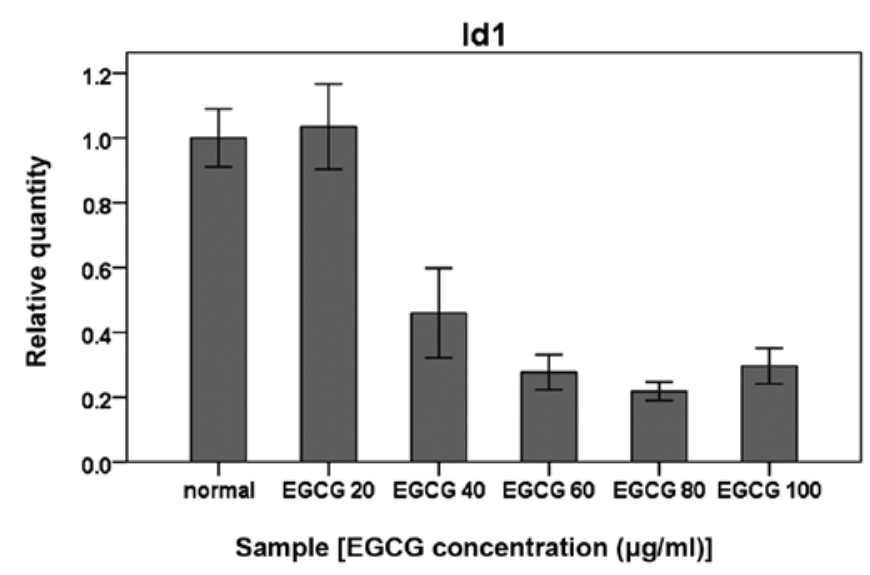

Figure 2. Gene expression microarray analysis of real-time RT-PCR showed that Id1 mRNA expression was significantly reduced in a concentration-dependent manner by EGCG. Id1 mRNA expression in AGS cells was significantly reduced after treatment with $80 \mu \mathrm{g} / \mathrm{ml}$ EGCG.

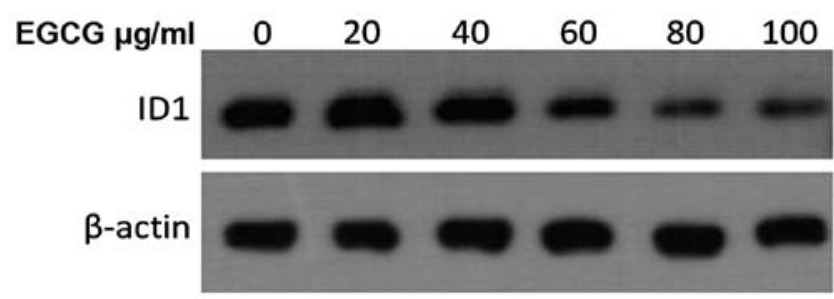

Figure 3. Western blot analysis showed that Id1 protein expression was significantly reduced in AGS cells treated with EGCG in a concentrationdependent manner. Id1 protein expression was significantly reduced after treatment with $80 \mu \mathrm{g} / \mathrm{ml}$ EGCG. $\beta$-actin was used as a loading control.

differentially expressed before and after the AGS gastric cancer cells were treated with EGCG.

Verification of microarray results with quantitative realtime RT-PCR and western blot analysis. To verify the results from gene expression microarray, the differentially expressed gene Id 1 was verified by quantitative real-time RT-PCR, using the primers listed in Table II. Id1 mRNA expression was significantly reduced in the AGS cells treated with EGCG in a concentration-dependent manner (Fig. 2, P<0.01). Western blot analysis further showed that Id1 protein expression in AGS cells was consistent with the mRNA expression (Fig. 3).

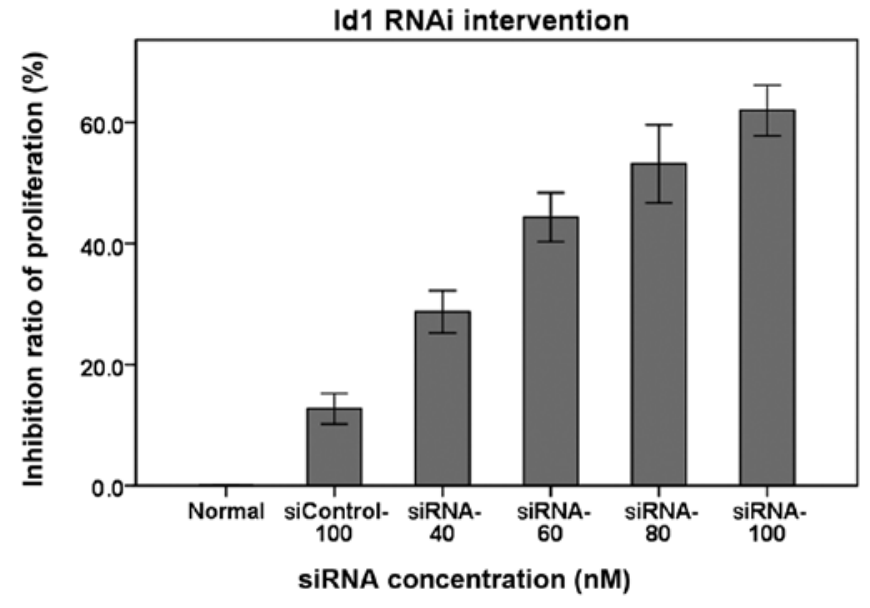

Figure 4. The CCK-8 experiment showed that Id1 RNAi affected AGS cell proliferation. The inhibition rates for cells treated with siRNA-Id1 at different concentrations and $100 \mathrm{nM}$ siRNA controls were significantly different $(\mathrm{P}<0.01)$. siRNA-Id1 inhibited proliferation of human AGS gastric cancer cells in a concentration-dependent manner. Proliferation of AGS cells treated with $80 \mathrm{nM}$ siRNA-Id1 was significantly inhibited, but there was no difference between cells treated with the same concentration of EGCG for 24 and $72 \mathrm{~h}(\mathrm{P}>0.05)$.

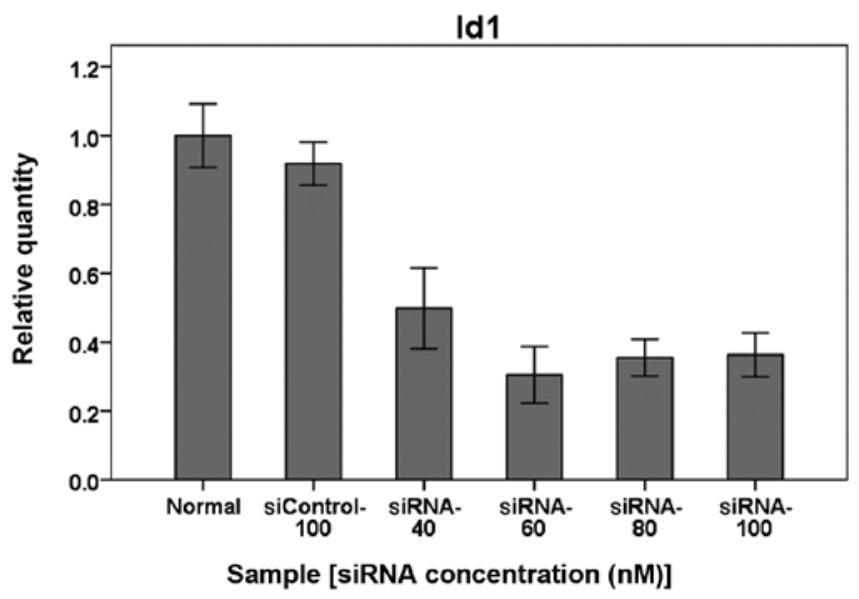

Figure 5. Real-time RT-PCR showed that Id1 mRNA expression in AGS cells treated with siRNA-Id1 was significantly reduced in a concentration-dependent manner. Id1 mRNA expression in AGS cells was significantly reduced after treatment with $80 \mu \mathrm{g} / \mathrm{ml}$ EGCG.

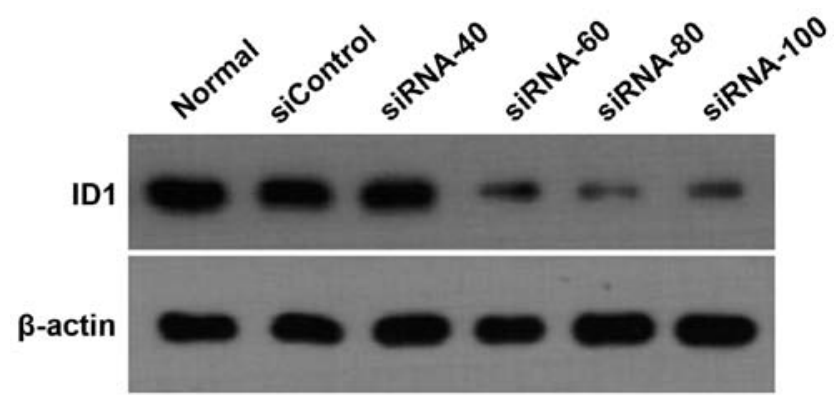

Figure 6. Western blot analysis showed that Id1 protein expression was significantly reduced in AGS cells treated with siRNA-Id1 in a concentrationdependent manner. $\beta$-actin was used as a loading control. 

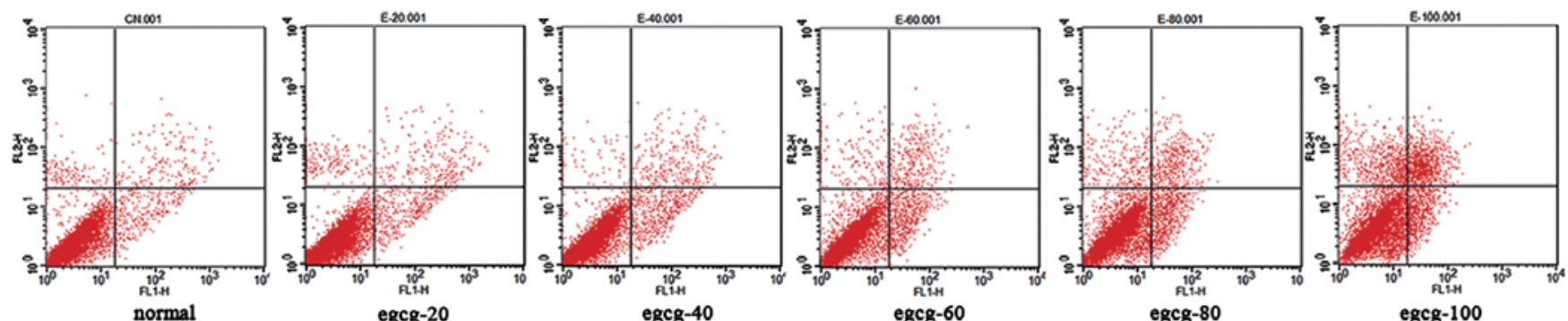

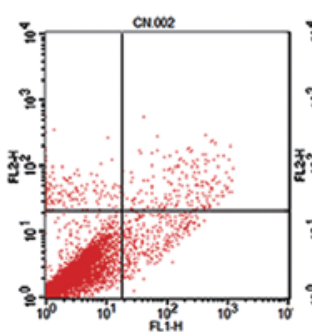

normal

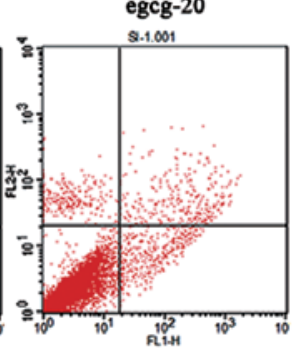

sicontrol-100
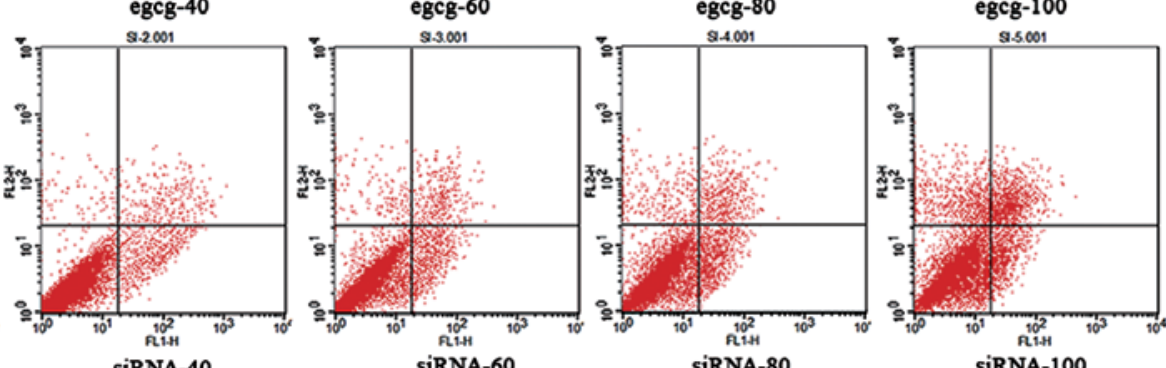

Figure 7. Effect of EGCG and Id1 RNAi on AGS gastric cancer cell apoptosis. Flow cytometry showed that EGCG and siRNA-Id1 concentration-dependently induced apoptosis of AGS cells. Apoptosis and necrosis gradually increased in AGS cells treated with different concentrations of EGCG. Similarly, apoptosis and necrosis also increased gradually in AGS cells treated with different concentrations of siRNA-Id1. No significant difference was found in the two groups for corresponding concentrations of EGCG and siRNA-Id1 ( $>>0.05)$. After treatment with Id1-RNAi, proliferation and apoptosis of AGS cells were similar to cells treated with EGCG.

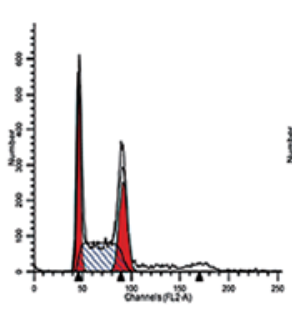

normal
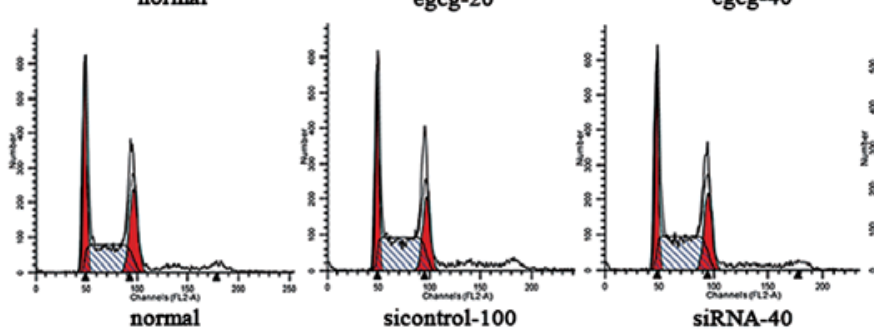

SiRNA-40

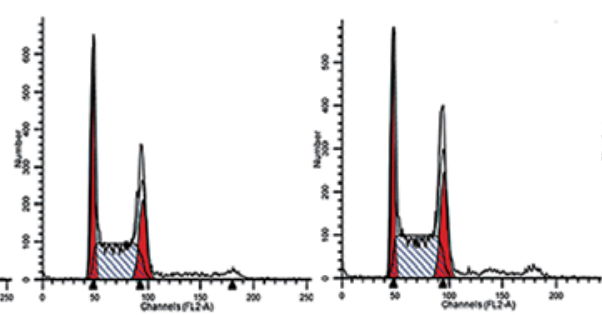

egcg-60

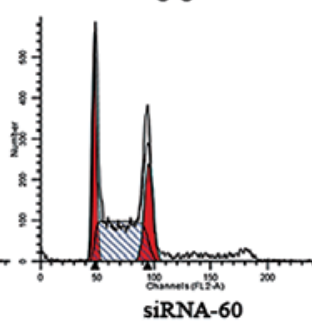

SiRNA-60

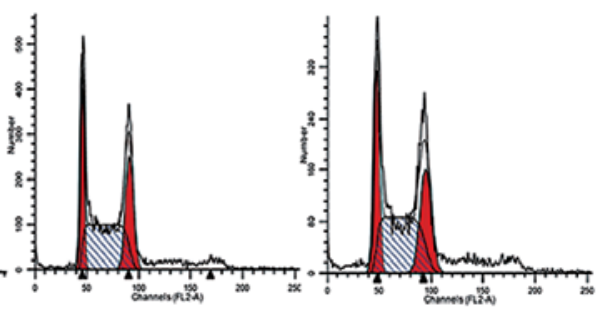

egcg-80
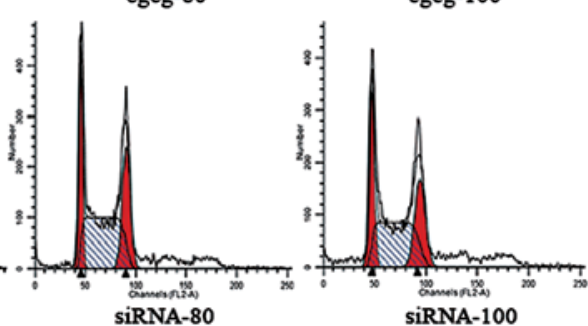

Figure 8. EGCG and Id1 RNAi affected the AGS cell cycle. AGS cells treated with EGCG and siRNA-Id1 were both arrested at $\mathrm{S}$ phase (P<0.05). Proliferation and apoptosis of AGS cells treated with Id1-RNAi were similar to the changes in cells treated with EGCG.

Detection of Idl with CCK-8 to observe influence of RNA $i$ on AGS cell proliferation. The CCK-8 experiment and cell morphological observation showed that siRNA-Id1 inhibited proliferation of AGS cells in a concentration-dependent manner (Fig. 4, P<0.01). Proliferation of AGS cells was significantly inhibited by $80 \mathrm{nM}$ siRNA-Id1.

Idl expression in AGS cells treated with IdI RNAi. Real-time RT-PCR showed that mRNA expression of Id1 was significantly downregulated in AGS cells treated with siRNA-Id1 in a concentration-dependent manner (Fig. 5, P<0.01). Western blot analysis further showed that Id1 protein expression in AGS cells was consistent with mRNA expression (Fig. 6).
Apoptosis and cell cycle of AGS gastric cancer cells treated with EGCG and IdI RNAi. Flow cytometry showed that EGCG and siRNA-Id1 induced apoptosis of AGS cells in a concentration-dependent manner (Fig. 7). After treatment with Id1-RNAi, changes in AGS cell proliferation and apoptosis were similar to those in cells treated with EGCG. AGS cells were arrested at $S$ phase after treatment with EGCG and siRNA-ID1 (Fig. 8, $\mathrm{P}<0.05$ ).

\section{Discussion}

An increasing number of studies have shown that EGCG regulates transduction of signaling molecules related to tumor 
cell metastasis and migration, and inhibits tumor cell proliferation and induces apoptosis and cell cycle arrest $(13,14)$. In the present study, CCK-8 experiments and morphological observation showed that EGCG inhibited proliferation of AGS gastric cancer cells in a concentration-dependent manner. Flow cytometry showed that EGCG concentration-dependently induced apoptosis of AGS cells and cell cycle arrest at S phase. We further used gene expression microarray analysis for initial screening of differentially expressed genes. There were 54 differentially expressed genes when comparing EGCG-treated and normal control cells, with 37 upregulated and 17 downregulated genes. Han et al have demonstrated that Id1 expression is often high in gastric cancer tissues and cell lines and its expression level is related to the degree of malignancy (15). Therefore, high Id1 expression is directly related to the malignant potential of gastric cancer cells. Tsuchiya et al have also found that gastric cancer cells with high Id1 expression have strong metastatic ability (16). As far as we know, the role of Id1 in EGCG-induced tumor inhibition has not been reported so far. Thus, we determined the differentially expressed Id1 gene by screening with gene expression microarray.

The Id protein family is a helix-loop-helix (HLH) family of transcription factors, including Id1-Id4. Id protein family members have a highly conserved HLH area, which can be combined with a basic HLH protein (bHLH) to form a heterodimer, thereby inhibiting the bHLH binding to target genes, reducing bHLH transcription factor activity, inhibiting cell differentiation and promoting cell proliferation $(17,18)$. Cell apoptosis and proliferation play an important role in the occurrence and development of malignant tumors (19). Id molecules can regulate cyclin-dependent kinase (cdk) inhibitors to shorten the cell cycle. Idl is directly involved in regulating the cell cycle by antisense oligonucleotides $(20,21)$ or microinjection of Id1 antibody (22) to inhibit partially Id1 protein expression, thus delaying cell entry into $\mathrm{S}$ phase. Idl promotes cdk4 and cdk2 to combine with retinoblastoma protein $(\mathrm{pRb})$, by inhibiting transcriptional level of $\mathrm{p} 16^{\mathrm{ink} 4 \mathrm{a}}$ and $\mathrm{p} 21^{\mathrm{WAF} 1}$, which catalyzes phosphorylation of $\mathrm{pRb}$. E2F and other types of protein can escape from $\mathrm{pRb}$ to activate more cancer gene transcription, so the cells enter into $S$ phase and become cancerous (23) and proliferate (24). In fibroblasts, Id1 activates the Ras-Raf-MEK pathway to promote cell proliferation (25). Ling et al (26) found that Id1 induces proliferation of prostate cancer cells by activating the mitogen-activated protein kinase pathway, which has a positive correlation with the degree of tumor cell malignancy (27). Tumor necrosis factor- $\alpha$-induced Id1 increases the activity of nuclear factor (NF)- $\mathrm{BB}$ and the anti-apoptotic effectors Bcl-xL and intercellular adhesion molecule-1, by inactivating the Bax and caspase-3 pathways, thus inhibiting apoptosis (28). Id-1 can also promote Bcl-2 expression and decrease expression of Bax and caspase- 3 by inhibiting the p53 signaling pathway and activating the $\mathrm{NF}-\kappa \mathrm{B}$ signaling pathway, thus preventing tumor cell apoptosis (29). Tsuchiya et al (16) reported that tumor cell proliferation and migration are significantly reduced in the Id1/Id3 double knockout gastric cancer cell line MKN 45. The number of peritoneal metastases is significantly reduced, and the size of single metastases is also decreased, which proves that Id1 and Id3 knockout can significantly inhibit peritoneal metastasis of gastric cancer cells. Therefore, Id might be a good target for treatment and prevention of gastric cancer.
After poorly differentiated AGS gastric cancer cells were treated with siRNA-Id1, proliferation slowed significantly, thus inhibiting Id1 mRNA and protein expression. Flow cytometry showed that apoptosis was increased and cells were arrested at $\mathrm{S}$ phase. Id1 RNAi affected the phenotypic changes in AGS gastric cancer cells in a dose-dependent manner. The above results suggest that Id1 plays its oncogenic role through promoting cancer cell proliferation and inhibiting apoptosis. Id1 participated in proliferation, apoptosis and other biological behavior of poorly differentiated AGS gastric cancer cells.

Previous studies have shown that EGCG can induce apoptosis through mitochondria $(30,31)$, p53 (32-34), Bcl-2 (35) cell signaling pathways $(36,37)$, reactive oxygen species (38) and telomerase (39). In different cell lines, EGCG can induce apoptosis through different mechanisms. We found that EGCG lowered Id1 expression to induce apoptosis and inhibit proliferation of poorly differentiated AGS gastric cancer cells, but it is necessary to investigate further how to achieve the above through a certain mechanism and whether the activation of signal transduction pathways is affected.

Molecular target therapy has become the trend in cancer treatment and research due to its high selectivity, good efficacy and few side-effects. Our experiments proved that EGCG could play a role in inhibiting proliferation, promoting apoptosis, and affecting cell cycle of poorly differentiated AGS gastric cancer cells, which is closely related to downregulation of Id 1 expression. Therefore, Id1 may be one of the targets regulated by EGCG for tumor inhibition.

\section{Acknowledgements}

Shanghai Municipal Health Bureau Key Disciplines Grant, no. ZK2012A05; National Natural Science Foundation, no. 81070344 .

\section{References}

1. Krejs GJ: Gastric cancer: epidemiology and risk factors. Dig Dis 28: 600-603, 2010.

2. Jemal A, Bray F, Center MM, Ferlay J, Ward E and Forman D: Global cancer statistics. CA Cancer J Clin 61: 69-90, 2011.

3. Yang L: Incidence and mortality of gastric cancer in China. World J Gastroenterol 12: 17-20, 2006.

4. Hemalswarya S and Doble M: Potential synergism of natural products in the treatment of cancer. Phytother Res 20: 239-249, 2006.

5. Cao G, Chen M, Song Q, et al: EGCG protects against UVB-induced apoptosis via oxidative stress and the JNK1/c-Jun pathway in ARPE19 cells. Mol Med Rep 5: 54-59, 2012.

6. Ziaedini A, Jafari A and Zakeri A: Extraction of antioxidants and caffeine from green tea (Camelia sinensis) leaves: kinetics and modeling. Food Sci Technol 16: 505-510, 2010.

7. Chen D, Wan SB, Yang H, Yuan J, Chan TH and Dou QP: EGCG, green tea polyphenols and their synthetic analogs and prodrugs for human cancer prevention and treatment. Adv Clin Chem 53: 155-177, 2011.

8. Rao SD and Pagidas K: Epigallocatechin-3-gallate, a natural polyphenol, inhibits cell proliferation and induces apoptosis in human ovarian cancer cells. Anticancer Res 30: 2519-2523, 2010.

9. Patra SK, Rizzi F, Silva A, Rugina DO and Bettuzzi S: Molecular targets of (-)-epigallocatechin-3-gallate (EGCG): specificity and interaction with membrane lipid rafts. J Physiol Pharmacol 59 (Suppl 9): 217-235, 2008.

10. Johnson JJ, Bailey HH and Mukhtar H: Green tea polyphenols for prostate cancer chemoprevention: a translational perspective. Phytomedicine 17: 3-13, 2010. 
11. Mandel SA, Amit T, Kalfon L, Reznichenko L, Weinreb O and Youdim MB: Cell signaling pathways and iron chelation in the neurorestorative activity of green tea polyphenols: special reference to epigallocatechin gallate (EGCG). J Alzheimers Dis 15: 211-222, 2008.

12. Yang H, Zonder JA and Dou QP: Clinical development of novel proteasome inhibitors for cancer treatment. Expert Opin Investig Drugs 18: 957-971, 2009.

13. Khan N and Mukhtar H: Multitargeted therapy of cancer by green tea polyphenols. Cancer Lett 269: 269-280, 2008.

14. Lin RW, Chen $\mathrm{CH}$, Wang YH, et al: (-)-Epigallocatechin gallate inhibition of osteoclastic differentiation via NF-kappaB. Biochem Biophys Res Commun 379: 1033-1037, 2009.

15. Han S, Gou C, Hong L, et al: Expression and significances of Id1 helix-loop-helix protein overexpression in gastric cancer. Cancer Lett 216: 63-71, 2004.

16. Tsuchiya T, Okaji Y, Tsuno NH, et al: Targeting Id1 and Id3 inhibits peritoneal metastasis of gastric cancer. Cancer Sci 96 784-790, 2005

17. Aranha MM, Sola S, Low WC, Steer CJ and Rodrigues CM Caspases and p53 modulate FOXO3A/Id1 signaling during mouse neural stem cell differentiation. J Cell Biochem 107: 748-758, 2009.

18. Benezra R, Davis RL, Lockshon D, Turner DL and Weintraub H: The protein Id: a negative regulator of helix-loop-helix DNA binding proteins. Cell 61: 49-59, 1990.

19. Evan $G$ and Littlewood T: A matter of life and cell death. Science 281: 1317-1322, 1998.

20. Barone MV, Pepperkok R, Peverali FA and Philipson L: Id proteins control growth induction in mammalian cells. Proc Natl Acad Sci USA 91: 4985-4988, 1994.

21. Hara E, Yamaguchi $\mathrm{T}$, Nojima $\mathrm{H}$, et al: Id-related genes encoding helix-loop-helix proteins are required for G1 progression and are repressed in senescent human fibroblasts. J Bio Chem 269: 2139-2145, 1994.

22. Peverali FA, Ramqvist T, Saffrich R, Pepperkok R, Barone MV and Philipson L: Regulation of G1 progression by E2A and Id helix-loop-helix proteins. EMBO J 13: 4291-4301, 1994.

23. Alani RM, Young AZ and Shifflett CB: Id1 regulation of cellular senescence through transcriptional repression of p16/Ink4a Proc Natl Acad Sci USA 98: 7812-7816, 2001.

24. Lee TK, Man K, Ling MT, et al: Over-expression of Id-1 induces cell proliferation in hepatocellular carcinoma through inactivation of p16INK4a/RB pathway. Carcinogenesis 24: 1729-1736, 2003.

25. Ohtani N, Zebedee Z, Huot TJ, et al: Opposing effects of Ets and Id proteins on p16INK4a expression during cellular senescence. Nature 409: 1067-1070, 2001.

26. Ling MT, Wang X, Ouyang XS, et al: Activation of MAPK signaling pathway is essential for Id-1 induced serum independent prostate cancer cell growth. Oncogene 21: 8498-8505, 2002 .
27. Cheung HW, Ling MT, Tsao SW, Wong YC and Wang X: Id-1-induced Raf/MEK pathway activation is essential for its protective role against taxol-induced apoptosis in nasopharyngeal carcinoma cells. Carcinogenesis 25: 881-887, 2004.

28. Ling MT, Wang X, Ouyang XS, Xu K, Tsao SW and Wong YC: Id-1 expression promotes cell survival through activation of NF-kappaB signalling pathway in prostate cancer cells. Oncogene 22: 4498-4508, 2003.

29. Kim H, Chung H, Kim HJ, et al: Id-1 regulates Bcl-2 and Bax expression through 553 and NF-kappaB in MCF-7 breast cancer cells. Breast Cancer Res Treat 112: 287-296, 2008.

30. Chen C, Shen G, Hebbar V, Hu R, Owuor ED and Kong AN: Epigallocatechin-3-gallate-induced stress signals in HT-29 human colon adenocarcinoma cells. Carcinogenesis 24 1369-1378, 2003

31. Qanungo S, Das M, Haldar S and Basu A: Epigallocatechin3-gallate induces mitochondrial membrane depolarization and caspase-dependent apoptosis in pancreatic cancer cells. Carcinogenesis 26: 958-967, 2005.

32. Gupta S, Ahmad N, Nieminen AL and Mukhtar H: Growth inhibition, cell-cycle dysregulation, and induction of apoptosis by green tea constituent (-)-epigallocatechin-3-gallate in androgen-sensitive and androgen-insensitive human prostate carcinoma cells. Toxicol Appl Pharmacol 164: 82-90, 2000.

33. Hofmann CS and Sonenshein GE: Green tea polyphenol epigallocatechin-3 gallate induces apoptosis of proliferating vascular smooth muscle cells via activation of p53. FASEB J 17: 702-704, 2003.

34. Roy AM, Baliga MS and Katiyar SK: Epigallocatechin-3gallate induces apoptosis in estrogen receptor-negative human breast carcinoma cells via modulation in protein expression of p53 and Bax and caspase-3 activation. Mol Cancer Ther 4: 81-90, 2005.

35. Nihal M, Ahmad N, Mukhtar H and Wood GS: Anti-proliferative and proapoptotic effects of (-)-epigallocatechin-3-gallate on human melanoma: possible implications for the chemoprevention of melanoma. Int J Cancer 114: 513-521, 2005.

36. Ahmad N, Gupta S and Mukhtar H: Green tea polyphenol epigallocatechin-3-gallate differentially modulates nuclear factor kappaB in cancer cells versus normal cells. Arch Biochem Biophys 376: 338-346, 2000.

37. Gupta S, Hastak K, Afaq F, Ahmad N and Mukhtar H: Essential role of caspases in epigallocatechin-3-gallate-mediated inhibition of nuclear factor kappa B and induction of apoptosis. Oncogene 23: 2507-2522, 2004.

38. Vittal R, Selvanayagam ZE, Sun Y, et al: Gene expression changes induced by green tea polyphenol (-)-epigallocatechin3 -gallate in human bronchial epithelial 21BES cells analyzed by DNA microarray. Mol Cancer Ther 3: 1091-1099, 2004.

39. Naasani I, Seimiya $\mathrm{H}$ and Tsuruo T: Telomerase inhibition, telomere shortening, and senescence of cancer cells by tea catechins. Biochem Biophys Res Commun 249: 391-396, 1998. 